\title{
Third-order perturbations of a zero-pressure cosmological medium: Pure general relativistic nonlinear effects
}

\author{
Jai-chan Hwang ${ }^{(a)}$ and Hyerim $\mathrm{Noh}^{(b)}$ \\ (a) Department of Astronomy and Atmospheric Sciences, Kyungpook National University, Taegu, Korea \\ (b) Korea Astronomy and Space Science Institute, Daejon, Korea
}

\begin{abstract}
We consider a general relativistic zero-pressure irrotational cosmological medium perturbed to the third order. We assume a flat Friedmann background but include the cosmological constant. We ignore the rotational perturbation which decays in expanding phase. In our previous studies we discovered that, to the second-order perturbation, except for the gravitational wave contributions, the relativistic equations coincide exactly with the previously known Newtonian ones. Since the Newtonian second-order equations are fully nonlinear, any nonvanishing third and higher order terms in the relativistic analyses are supposed to be pure relativistic corrections. In this work we derive such correction terms appearing in the third order. Continuing our success in the second-order perturbations we take the comoving gauge. We discover that the third-order correction terms are of $\varphi_{v}$-order higher than the second-order terms where $\varphi_{v}$ is a gauge-invariant combination related to the three-space curvature perturbation in the comoving gauge; compared with the Newtonian potential we have $\delta \Phi \sim \frac{3}{5} \varphi_{v}$ to the linear order. Therefore, the pure general relativistic effects are of $\varphi_{v}$-order higher than the Newtonian ones. The corrections terms are independent of the horizon scale and depend only on the linear order gravitational potential (curvature) perturbation strength. From the temperature anisotropy of cosmic microwave background we have $\frac{\delta T}{T} \sim \frac{1}{3} \delta \Phi \sim \frac{1}{5} \varphi_{v} \sim$ $10^{-5}$. Therefore, our present result reinforces our previous important practical implication that near current era one can use the large-scale Newtonian numerical simulation more reliably even as the simulation scale approaches near (and goes beyond) the horizon.
\end{abstract}

\section{INTRODUCTION}

In our previous works $[1,2]$ we have proved that in the zero-pressure irrotational cosmological medium the relativistic second-order scalar-type perturbation equations coincide exactly with the known ones in Newtonian theory. This result shows a continuation of the relativistic-Newtonian correspondences of the cosmological zero-pressure medium previously shown in the background world model by Friedmann in 1922 [3] and by Milne in 1934 [4], and in the linear perturbation by Lifshitz in 1946 [5] and by Bonnor in 1957 [6]. History shows that both for the background and for the linear perturbation the equations were first derived in Einstein's gravity $[3,5]$ and later followed by studies in Newton's gravity $[4,6]$. In the case of second-order perturbations the Newtonian result was known first [7]. Since the Newtonian results are not supposed to be reliable as the scale approaches the horizon, our result has a practical importance by showing that even to the second-order perturbations we can use Newtonian equations in all scales. In this work we will extend the situation to the next order in relativistic perturbation which, as we will explain shortly, can be regarded as pure relativistic corrections. Even in the linear perturbations the presence of the tensor-type perturbation (gravitational waves) can be regarded as a pure relativistic effect. To the second order, additionally, the gravitational waves and the scalar-type perturbation are coupled, thus one can source the other. This can be regarded as another pure relativistic correction.
In this work we will derive pure relativistic corrections of the scalar-type perturbation which appear in the thirdorder perturbations. The situation we have is that to the second order Einstein's theory gives exactly the same result as the Newton's theory whereas in the Newtonian case such second-order equations are in fact exactly valid to fully nonlinear order. Therefore, any nonvanishing third and higher order perturbations in Einstein's gravity are supposed to be the pure relativistic corrections. We will present the scalar-type perturbation equations to the third order and the gravitational wave equation to the second order. This is enough because the previously known gravitational wave equation will already get the correction terms even in the second order, whereas for the scalar-type perturbation new correction terms appear only in the third order compared with the previous studies [2]. If we include the gravitational waves, the gravitational waves only to the second order will be needed to make the third-order scalar type perturbation equations complete.

We will ignore the vector-type perturbation in this work, thus consider only irrotational perturbation, because due to the angular momentm conservation the rotational perturbation always decays in expanding phase; this situation will be reversed in the collapsing phase where the rotational mode will grow again due to the angular momentm conservation. We will consider a flat background with two reasons. First, even in the linear perturbations it is known that the relativistic-Newtonian correspondence is rather ambiguous in the presence of the 
background curvature; this is the case if we include the gravitational potential in making the correspondence [8]. Second, current observations of the large-scale structure and the cosmic microwave background radiation favour near flat Friedmann world model with non-vanishing cosmological constant [9]. In fact, we will include the cosmological constant in our analyses, thus our results are relevant to currently favoured cosmology.

Our previous study on the second-order perturbations revealed which gauge condition (equivalently, gaugeinvariant combinations) suits our problem [2]. In fact, unique gauge conditions were distinguished in showing the correspondence: these were the spatial $C$-gauge and the temporal comoving gauge. The spatial $C$-gauge was a rather natural choice because only in this gauge the spatial gauge mode can be fixed completely to all orders. In this gauge all the rest of the variables can be equivalently regarded as spatially gauge-invariant ones to all orders, see [1]. In the temporal gauge choice, however, we have many (in fact, infinitely many) different gauge choices, which fix the temporal gauge mode completely. In any of such gauge conditions all the rest of the variables can be equivalently regarded as temporally gauge-invariant ones. Such procedures for taking the temporal gauge condition are separately available to each order in perturbations; i.e., we can choose different gauge conditions to different perturbational order, see [1].

The (temporal) comoving gauge distinguished itself with the following reasons. In [2] we have successfully shown that in the comoving gauge we can identify the density and velocity variables which allow us to derive relativistic equations identical to the Newtonian ones. This point, perhaps, does not necessarily imply that it is not possible to discover any other variables and gauges which also lead to the same identification. But, we do mean that the comoving gauge is a natural choice. This also does not necessarily imply that even to the third order the comoving gauge choice will be the best choice. As a matter of fact, our policy/strategy about the gauge choice is that we do not know which gauge will suit the problem before we investigate and try many different gauge conditions. That is why we have presented our basic set of equations in a gauge-ready form which allows maximal usage of many different gauge conditions: see $[10,11]$ for the linear case and [1] for the second-order perturbations.

In this work, based on our successful experiences in the linear and second-order perturbations we will take the temporal comoving gauge condition and the spatial $C$-gauge even to the third order. First, under this gauge condition we can write the fully nonlinear equations in simple forms. Second, only in this gauge we have the relativistic equations the same as the Newtonian ones to the second order. And third, quite interestingly, we will show that in order to derive the third-order perturbations in this gauge, in fact, we need to evaluate the geometric and energy-momentum variables to the second order only. This is possible due to our appropriate choice of the variables and the gauge conditions. Thus, in the following we will derive the third-order pure relativistic correction terms in the comoving gauge condition. As we mentioned, since our gauge conditions fix the gauge modes completely, each of the variables we are using has a unique corresponding gauge-invariant combination. Thus, our analyses can be equivalently regarded as gauge-invariant ones extended to the third-order perturbations.

Here, we summarize the relativistic-Newtonian correspondences known up to second-order perturbations in a flat Friedmann world model without pressure. To the background order we have $[3,4]$

$$
H^{2}=\frac{8 \pi G}{3} \mu-\frac{\text { const. }}{a^{2}}+\frac{\Lambda}{3},
$$

with the energy (mass) density $\mu(\varrho) \propto a^{-3} ; a(t)$ is the cosmic scale factor and $H \equiv \dot{a} / a$; we set $c \equiv 1$. The "const." part is interpreted as the spatial curvature in Einstein's gravity [3], and the total energy in the Newton's gravity [4]. We put the cosmological constant $\Lambda$ by hand which can work as a repulsive force proportional to the distance for $\Lambda>0$. To the linear-order perturbations we have $[5,6]$

$$
\ddot{\delta}+2 H \dot{\delta}-4 \pi G \varrho \delta=0,
$$

where $\delta \equiv \delta \mu / \mu=\delta \varrho / \varrho$ with $\mu(\varrho)$ and $\delta \mu(\delta \varrho)$ the background and perturbed parts of the energy (mass) density. This equation is valid considering general presence of the background spatial curvature term and the cosmological constant. Now, to the second order, assuming flat background, we have $[7,1,2]$

$$
\begin{aligned}
\dot{\delta}+\frac{1}{a} \nabla \cdot \mathbf{u} & =-\frac{1}{a} \nabla \cdot(\delta \mathbf{u}), \\
\nabla \cdot(\dot{\mathbf{u}}+H \mathbf{u})+4 \pi G \varrho a \delta & =-\frac{1}{a} \nabla \cdot(\mathbf{u} \cdot \nabla \mathbf{u}),
\end{aligned}
$$

or by combining these, we have

$$
\begin{aligned}
\ddot{\delta}+ & 2 H \dot{\delta}-4 \pi G \varrho \delta \\
& =-\frac{1}{a^{2}} \frac{\partial}{\partial t}[a \nabla \cdot(\delta \mathbf{u})]+\frac{1}{a^{2}} \nabla \cdot(\mathbf{u} \cdot \nabla \mathbf{u}) .
\end{aligned}
$$

These equations are valid in the presence of cosmological constant. The above equations are valid in both Einstein's and Newton's theories. In the relativistic theory we have to specify the variables $\delta$ and $\mathbf{u}$ which correspond to the relative density fluctuation and perturbed velocity in Newton's theory; this will be done later.

We believe no one would have anticipated such an exact coincidence to the second-order perturbations especially considering the presence of the horizon in the relativistic treatment. It might happen as well that our relativistic results give relativistic correction terms appearing to the second order which could become important as the scale approaches and goes beyond the horizon. Our results show that there are no such correction terms 
appearing to the second order, and the correspondence is exact to that order. Equations (3)-(5) are valid in fully nonlinear situation in Newton's theory [7] whereas these are valid only up to the second order in Einstein's case $[1,2]$. It is our task to derive the third-order correction terms in Einstein's theory and to show how it causes difference between the two theories.

\section{FULLY NONLINEAR EQUATIONS}

In [2] we have presented the fully nonlinear equations in the comoving gauge condition using the $1+3$ covariant formulation [12] and the $3+1$ ADM (Arnowitt-DeserMisner) [13] formulations of Einstein's gravity. In [2] we showed that these two formulations are equivalent, and in the following we will take the ADM formulation. The basic set of ADM equations in our notation can be found in §II.A of [1], see also [14]. As this work can be regarded as a continuation of our previous studies in $[1,2]$, we follow the notations used in that works.

In the ADM approach, the temporal comoving gauge condition to all orders sets the flux four-vector to vanish, i.e., $J_{\alpha} \equiv 0$; here we also used the irrotational condition which ignores the vector-type perturbation. In [1] the fluid quantities are introduced based on the normal frame four-vector $\tilde{n}_{a}$ with $\tilde{n}_{\alpha} \equiv 0$; in this case the information of the fluid motion is present in the flux vector $\tilde{q}_{a}$ with $\tilde{q}_{a} \tilde{n}^{a} \equiv 0$. In such a choice of the frame the temporal comoving gauge condition with vanishing rotation implies $\tilde{u}_{\alpha} \equiv 0$ of the fluid four-vector $\tilde{u}_{a}$. Thus, the fluid fourvector coincides with the normal four-vector. The physical zero-pressure condition implies vanishing isotropic pressure ( $\tilde{p}$ or $S$ in the ADM notation) and anisotropic stress $\left(\tilde{\pi}_{a b}\right.$ or $\left.\bar{S}_{\alpha \beta}\right)$, i.e., $\tilde{p} \equiv 0 \equiv \tilde{\pi}_{a b}$ or $S \equiv 0 \equiv \bar{S}_{\alpha \beta}$ to all orders, based on the fluid four-vector. As the $\tilde{u}_{a}$ coincides with the $\tilde{n}_{a}$ in our comoving gauge, the zeropressure conditions simply allow us to set all the pressure terms in the normal-frame fluid quantites equal to zero; we point out that this is not true in the other gauge conditions in the normal-frame fluid quantities, see [2]; in the normal frame, in other than the comoving gauge conditions the pressure and anisotropic stress do not vanish to the second and higher orders even in the physically zero-pressure situation; for further discussions, see [2]. Although we do not need the form of energy-momentum tensor, from Eqs. (3), (4) of [1] we have

$$
\tilde{T}^{00}=N^{-2} E, \quad \tilde{T}_{\alpha}^{0}=0, \quad \tilde{T}_{\alpha \beta}=0,
$$

where tildes indicate the covariant quantities; $E$ is the ADM energy density and $N$ is the lapse function defined as $N^{2} \equiv-\left(\tilde{g}^{00}\right)^{-1}$.

The momentum conservation equation in Eq. (13) of [1] gives

$$
N_{, \alpha}=0
$$

Thus, we may set $N \equiv a(t)$ to all orders. In this case we have $\dot{E} \equiv E_{0_{0}} N^{-1}$. The energy conservation equation and the trace part of ADM propagation equation in Eqs. (12), (10) of [1] give

$$
\begin{aligned}
\hat{\dot{E}}-K E & =0, \\
\hat{\dot{K}}-\frac{1}{3} K^{2}-\bar{K}^{\alpha \beta} \bar{K}_{\alpha \beta}-4 \pi G E+\Lambda & =0,
\end{aligned}
$$

where $\hat{\dot{E}} \equiv \dot{E}-E_{, \alpha} N^{\alpha} N^{-1}$, etc.; $K$ and $\bar{K}_{\alpha \beta}$ are the trace and tracefree parts, respectively, of the extrinsic curvature $K_{\alpha \beta}$ of the normal hypersurface introduced in the ADM formulation, and $N_{\alpha}$ is a shift vector defined as $N_{\alpha} \equiv \tilde{g}_{0 \alpha}$. The spatial indices in the ADM formulation are based on the ADM three-space metric $h_{\alpha \beta}$ defined as $h_{\alpha \beta} \equiv \tilde{g}_{\alpha \beta}$. By combining these equations we have

$$
\left(\frac{\hat{\dot{E}}}{E}\right)^{\hat{E}}-\frac{1}{3}\left(\frac{\hat{\dot{E}}}{E}\right)^{2}-\bar{K}^{\alpha \beta} \bar{K}_{\alpha \beta}-4 \pi G E+\Lambda=0 .
$$

Equations (6)-(10) are valid to all orders, i.e., these equations are fully nonlinear.

\section{THIRD ORDER PERTURBATIONS}

We consider the scalar- and tensor-type perturbations in the flat Friedmann background. As the metric we take

$$
\begin{aligned}
d s^{2}= & -a^{2}(1+2 \alpha) d \eta^{2}-2 a^{2} \beta_{, \alpha} d \eta d x^{\alpha} \\
& +a^{2}\left[g_{\alpha \beta}^{(3)}(1+2 \varphi)+2 \gamma_{, \alpha \mid \beta}+2 C_{\alpha \beta}^{(t)}\right] d x^{\alpha} d x^{\beta},
\end{aligned}
$$

where $\alpha, \beta, \gamma$ and $\varphi$ are spacetime dependent perturbedorder variables; we take Bardeen's metric convention in $[10,11]$ extended to the third order. A vertical bar indicates a covariant derivative based on $g_{\alpha \beta}^{(3)}$ which can be regarded as $\delta_{\alpha \beta}$ if we use Cartesian coordinates in the flat Friedmann background. By taking $\gamma \equiv 0$, which we call the spatial $C$-gauge, the spatial gauge mode is removed completely, thus all the remaining variables we are using are spatially gauge-invariant to the third order; this is true if we simultaneously take a temporal gauge which removes the temporal gauge mode completely, see $\S$ VI.B. 2 and C.1 of [1]. In the following we will take $\gamma \equiv 0$ as the spatial gauge condition and use $\chi \equiv a \beta+a^{2} \dot{\gamma}$ which becomes $\chi=a \beta$.

We expand

$$
E \equiv \mu+\delta \mu, \quad K \equiv-3 H+\kappa .
$$

Up to this point our notations look exactly the same as in the linear theory whereas, in fact, we consider each perturbation variable to be expanded to the third order. As an example, for $\delta \mu$ we have

$$
\delta \mu \equiv \delta \mu^{(1)}+\delta \mu^{(2)}+\delta \mu^{(3)}+\ldots,
$$


where, to the third order we truncate the expansion at third-order term $\delta \mu^{(3)}$. A close examination of our fully nonlinear equations in $\S I I$ reveals an important technical methods of performing the third-order perturbations by using only the second-order expansion of perturbation variables. Such a simple method is possible due to our right choice of the equations, the right gauge conditions, and our proper choice of the fundamental variables to be matched with the Newtonian variables to the third order. That is, in our calculations we do not even need to have the inverse metric expanded to the third order, and all algebraic quantities we need can be found in [1] which presents various useful quantities expanded to the second order in perturbations.

To the linear order we identified [8]

$$
\begin{aligned}
& \delta \varrho \equiv \delta \mu_{v}, \quad \delta \Phi \equiv-\varphi_{\chi}=\alpha_{\chi}, \\
& \mathbf{u} \equiv-\nabla v_{\chi}, \quad-\frac{1}{a} \nabla \cdot \mathbf{u} \equiv \frac{\Delta}{a} v_{\chi} \equiv \kappa_{v} .
\end{aligned}
$$

To the second order we identified [2]

$$
\delta \mu_{v} \equiv \delta \varrho, \quad \kappa_{v} \equiv-\frac{1}{a} \nabla \cdot \mathbf{u} .
$$

Based on our experience in the second-order perturbations, we have in mind to identify Eq. (15) even to the third order. It may turn out to be that these are not the best identifications, but in the following we will assume these are the right ones and will take the consequent additional third-order terms as the pure relativistic corrections.

The perturbed-order variable $v$ is defined as $J_{\alpha} \equiv$ $-a \mu v_{, \alpha}$ to all orders, thus $v$ is related to the velocity or flux variable. Our comoving gauge condition sets $v \equiv 0$. In our notation, $\delta \mu_{v}$ indicates a gauge-invariant combination which is the same as $\delta \mu$ in the comoving gauge which sets $v \equiv 0$. Such a variable (equivalently, a gaugeinvariant combination) is unique to all orders in perturbations; to the linear order we have $\delta \mu_{v} \equiv \delta \mu-\dot{\mu} a v$, and to the second order see Eq. (282) in [1]. Similarly, $\varphi_{\chi}$ is a gauge-invariant combination equivalent to $\varphi$ in the zeroshear gauge which sets $\chi \equiv 0$; to the linear order we have $\varphi_{\chi} \equiv \varphi-H \chi$, and to the second order, see Eq. (280) in [1]. For our justification to name the gauge conditions, see below Eq. (265) of [1].

One of the terms we need to evaluate to the third order in Eqs. (8)-(10) is $\bar{K}^{\alpha \beta} \bar{K}_{\alpha \beta}$. Since $\bar{K}_{\alpha \beta}$ is already at least linear order, we need to evaluate $\bar{K}^{\alpha \beta}$ to the second order only, etc. The other ones we need to evaluate to the third order are

$$
\hat{\dot{E}} \equiv \dot{E}-E_{, \alpha} N^{\alpha} N^{-1}, \quad \hat{\dot{K}} \equiv \dot{K}-K_{, \alpha} N^{\alpha} N^{-1} .
$$

Due to our identifications in Eq. (15), we are using the perturbed parts of $E$ and $K$ in Eq. (12) as the fundamental perturbation variables. In order to evaluate $E_{, \alpha} N^{\alpha}$, since $E_{, \alpha}\left(\right.$ or $\left.N^{\alpha}\right)$ is already at least linear order, it is enough to evaluate $N^{\alpha}$ (or $E_{, \alpha}$ ) only to the second order, and similarly for $K_{, \alpha} N^{\alpha}$. Thus, using Eqs. (55), (57), and (175) of [1], we have

$$
\begin{gathered}
E_{, \alpha} N^{\alpha}=-\frac{1}{a} \delta \mu_{, \alpha} \chi^{, \alpha}(1-2 \varphi)+2 \frac{1}{a} \delta \mu^{, \alpha} \chi^{, \beta} C_{\alpha \beta}^{(t)} \\
K_{, \alpha} N^{\alpha}=-\frac{1}{a} \kappa_{, \alpha} \chi^{, \alpha}(1-2 \varphi)+2 \frac{1}{a} \kappa^{, \alpha} \chi^{, \beta} C_{\alpha \beta}^{(t)} \\
\bar{K}^{\alpha \beta} \bar{K}_{\alpha \beta}=\left[\frac{1}{a^{2}}\left(\chi^{, \alpha \mid \beta}-\frac{1}{3} g^{(3) \alpha \beta} \Delta \chi\right)+\dot{C}^{(t) \alpha \beta}\right] \\
\times\left\{\left(\frac{1}{a^{2}} \chi_{, \alpha \mid \beta}+\dot{C}_{\alpha \beta}^{(t)}\right)(1-2 \alpha-4 \varphi)-\frac{4}{a^{2}} \chi_{, \alpha} \varphi, \beta\right. \\
-4 \dot{\varphi} C_{\alpha \beta}^{(t)}-\frac{2}{a^{2}} \chi^{, \gamma}\left(2 C_{\gamma \alpha \mid \beta}^{(t)}-C_{\alpha \beta \mid \gamma}^{(t)}\right) \\
\left.-4 C_{\alpha}^{(t) \gamma}\left(\frac{1}{a^{2}} \chi_{, \beta \mid \gamma}+\dot{C}_{\beta \gamma}^{(t)}\right)\right\} .
\end{gathered}
$$

Thus, Eqs. (8), (9) give

$$
\begin{aligned}
\left(\frac{\dot{\mu}}{\mu}+\right. & 3 H)(1+\delta)+\dot{\delta}-\kappa \\
& =\kappa \delta-\frac{1}{a^{2}} \delta_{, \alpha} \chi^{, \alpha}(1-2 \varphi)+2 \frac{1}{a^{2}} \delta^{, \alpha} \chi^{, \beta} C_{\alpha \beta}^{(t)}, \\
-3 \dot{H} & -3 H^{2}-4 \pi G \mu+\Lambda+\dot{\kappa}+2 H \kappa-4 \pi G \delta \mu \\
& =\frac{1}{3} \kappa^{2}-\frac{1}{a^{2}} \kappa_{, \alpha} \chi^{, \alpha}(1-2 \varphi)+2 \frac{1}{a^{2}} \kappa^{, \alpha} \chi^{, \beta} C_{\alpha \beta}^{(t)} \\
& +\left[\frac{1}{a^{2}}\left(\chi^{, \alpha \mid \beta}-\frac{1}{3} g^{(3) \alpha \beta} \Delta \chi\right)+\dot{C}^{(t) \alpha \beta}\right] \\
& \times\left[\left(\frac{1}{a^{2}} \chi_{, \alpha \mid \beta}+\dot{C}_{\alpha \beta}^{(t)}\right)(1-2 \alpha-4 \varphi)\right. \\
& -\frac{4}{a^{2}} \chi_{, \alpha} \varphi_{, \beta}-4 \dot{\varphi} C_{\alpha \beta}^{(t)}-\frac{2}{a^{2}} \chi^{, \gamma}\left(2 C_{\gamma \alpha \mid \beta}^{(t)}-C_{\alpha \beta \mid \gamma}^{(t)}\right) \\
& \left.-4 C_{\alpha}^{(t) \gamma}\left(\frac{1}{a^{2}} \chi_{, \beta \mid \gamma}+\dot{C}_{\beta \gamma}^{(t)}\right)\right] .
\end{aligned}
$$

We note that $\alpha$ and $\dot{\varphi}$ in the comoving gauge are already quadratic order at least, thus can be ignored in Eq. (21); see Eqs. (12), (20) of [2].

Now, we need Newtonian expressions of $\varphi$ to the linear order and $\chi$ to the second order. Since we are considering the comoving gauge condition our $\varphi$ and $\chi$ are the same as the gauge-invariant combinations $\varphi_{v}$ and $\chi_{v}$, respectively. To the linear order we have

$$
\begin{aligned}
& \varphi_{v} \equiv \varphi-a H v=\varphi_{\chi}-a H v_{\chi} \\
& \chi_{v} \equiv \chi-a v \equiv-a v_{\chi} .
\end{aligned}
$$

To the second order, $\chi_{v}$ is presented in Eq. (284) of [1]. To the second order, from Eq. (197) of [1] we have

$$
\begin{aligned}
\kappa+ & \frac{\Delta}{a^{2}} \chi=\left.N_{2}^{(s)}\right|_{v}=\frac{1}{a^{2}}\left(2 \varphi \Delta \chi-\chi^{, \alpha} \varphi_{, \alpha}\right) \\
& +\frac{3}{2} \frac{1}{a^{2}} \Delta^{-1} \nabla^{\alpha}\left(\chi_{, \alpha} \Delta \varphi+\chi^{, \beta} \varphi_{, \alpha \mid \beta}\right)+\frac{2}{a^{2}} \chi^{, \beta \mid \gamma} C_{\beta \gamma}^{(t)}
\end{aligned}
$$




$$
\begin{aligned}
& +\frac{3}{2} \Delta^{-1} \nabla^{\alpha}\left(-\varphi^{, \beta} \dot{C}_{\alpha \beta}^{(t)}+\frac{1}{a^{2}} \chi^{, \beta} \Delta C_{\alpha \beta}^{(t)}\right. \\
& \left.+2 C^{(t) \beta \gamma} \dot{C}_{\alpha \beta \mid \gamma}^{(t)}-C^{(t) \beta \gamma} \dot{C}_{\beta \gamma \mid \alpha}^{(t)}\right)+\frac{1}{2} C^{(t) \beta \gamma} \dot{C}_{\beta \gamma}^{(t)} \\
& \equiv \frac{1}{a} X
\end{aligned}
$$

where we have ignored $\alpha$ and $\dot{\varphi}$ which contribute to the third order. Apparently, we also need $\dot{C}_{\alpha \beta}^{(t)}$ to the second order. This will be presented in $\S \mathrm{V}$.

\section{SCALAR-TYPE CORRECTIONS}

Ignoring $C_{\alpha \beta}^{(t)}$, the perturbed parts of Eqs. (20), (21), and (23) give the complete set. As we ignore the rotational type perturbation the Newtonian velocity perturbation $\mathbf{u}$ is of a potential type, i.e., $\mathbf{u}=\nabla u$. Thus, Eqs. (15), (23) give

$$
\kappa \equiv-\frac{1}{a} \nabla \cdot \mathbf{u}=-\frac{1}{a} \Delta u, \quad \chi=a u+a \Delta^{-1} X,
$$

where $\kappa$ is valid to the third order and $\chi$ is valid to the second order. Thus, Eqs. (20), (21), and (23) can be written as

$$
\begin{aligned}
& \dot{\delta}+\frac{1}{a} \nabla \cdot \mathbf{u}=-\frac{1}{a} \nabla \cdot(\delta \mathbf{u}) \\
&+\frac{1}{a}\left[2 \varphi \mathbf{u}-\nabla\left(\Delta^{-1} X\right)\right] \cdot \nabla \delta, \\
& \frac{1}{a} \nabla \cdot(\dot{\mathbf{u}}+H \mathbf{u})+4 \pi G \mu \delta=-\frac{1}{a^{2}} \nabla \cdot(\mathbf{u} \cdot \nabla \mathbf{u}) \\
& \quad-\frac{2}{3 a^{2}} \varphi \mathbf{u} \cdot \nabla(\nabla \cdot \mathbf{u})+\frac{4}{a^{2}} \nabla \cdot\left[\varphi\left(\mathbf{u} \cdot \nabla \mathbf{u}-\frac{1}{3} \mathbf{u} \nabla \cdot \mathbf{u}\right)\right] \\
& \quad-\frac{\Delta}{a^{2}}\left[\mathbf{u} \cdot \nabla\left(\Delta^{-1} X\right)\right]+\frac{1}{a^{2}} \mathbf{u} \cdot \nabla X+\frac{2}{3 a^{2}} X \nabla \cdot \mathbf{u},
\end{aligned}
$$

where

$$
X \equiv 2 \varphi \nabla \cdot \mathbf{u}-\mathbf{u} \cdot \nabla \varphi+\frac{3}{2} \Delta^{-1} \nabla \cdot[\mathbf{u} \cdot \nabla(\nabla \varphi)+\mathbf{u} \Delta \varphi]
$$

Equations (25), (26) extend Eqs. (3), (4) to the third order. By combining Eqs. (25), (26) we can derive

$$
\begin{aligned}
\ddot{\delta} & +2 \frac{\dot{a}}{a} \dot{\delta}-4 \pi G \mu \delta=-\frac{1}{a^{2}} \frac{\partial}{\partial t}[a \nabla \cdot(\delta \mathbf{u})]+\frac{1}{a^{2}} \nabla \cdot(\mathbf{u} \cdot \nabla \mathbf{u}) \\
& +\frac{1}{a^{2}} \frac{\partial}{\partial t}\left\{a\left[2 \varphi \mathbf{u}-\nabla\left(\Delta^{-1} X\right)\right] \cdot \nabla \delta\right\} \\
& +\frac{2}{3 a^{2}} \varphi \mathbf{u} \cdot \nabla(\nabla \cdot \mathbf{u})-\frac{4}{a^{2}} \nabla \cdot\left[\varphi\left(\mathbf{u} \cdot \nabla \mathbf{u}-\frac{1}{3} \mathbf{u} \nabla \cdot \mathbf{u}\right)\right] \\
& +\frac{\Delta}{a^{2}}\left[\mathbf{u} \cdot \nabla\left(\Delta^{-1} X\right)\right]-\frac{1}{a^{2}} \mathbf{u} \cdot \nabla X-\frac{2}{3 a^{2}} X \nabla \cdot \mathbf{u},
\end{aligned}
$$

which extends Eq. (5) to the third order. The last three lines of Eq. (28) are the third-order terms. Examination of Eqs. (25)-(27) shows that all the third-order correction terms are of $\varphi$-order higher than the second-order terms like $\frac{1}{a} \nabla \cdot(\delta \mathbf{u})$ and $\frac{1}{a^{2}} \nabla \cdot(\mathbf{u} \cdot \nabla \mathbf{u})$. As the fully nonlinear zero-pressure Newtonian equations are exact to the second order, the above third-order correction terms in our relativistic analyses are pure relativistic correction terms. Therefore, the pure general relativistic effects are at least $\varphi$-order higher than the Newtonian ones. Up to the third-order corrections appearing in the general relativity, the effects are independent of the horizon scale and depend on the linear-order curvature (gravitational potential, see below) perturbation strength only.

In Eqs. (25)-(27) we need $\varphi$ only to the linear order. Thus, let us examine the behavior of $\varphi$ to the linear order. In our comoving gauge condition $\varphi$ is equivalent to a gauge-invariant combination $\varphi_{v}$, and to the linear order from Eq. (22) we have

$$
\varphi_{v}=\varphi_{\chi}-a H v_{\chi}
$$

where we have $\varphi_{\chi}=-\delta \Phi$ and $\mathbf{u}=-\nabla v_{\chi}$ in Eq. (14). Thus, in terms of the Newtonian variables we have

$$
\varphi=-\delta \Phi+\dot{a} \Delta^{-1} \nabla \cdot \mathbf{u}
$$

Exact solutions of linear perturbation were presented in Tables of [15]. For $K=0$ we have [15]

$$
\begin{aligned}
& \varphi_{v}=C, \\
& \varphi_{\chi}=-\alpha_{\chi}=-4 \pi G \mu a^{2} \Delta^{-1} \delta_{v}=C 4 \pi G \mu a^{2} H \int^{t} \frac{d t}{\dot{a}^{2}}, \\
& v_{\chi}=a \Delta^{-1} \kappa_{v}=-C \frac{1}{a H}\left(1+a^{2} H \dot{H} \int^{t} \frac{d t}{\dot{a}^{2}}\right),
\end{aligned}
$$

where the lower bounds of integrations give decaying modes. For $K=0=\Lambda$, using $a \equiv a_{1} t^{2 / 3}$, we have [15]

$$
\begin{aligned}
& \varphi_{v}=C \\
& \varphi_{\chi}=-\alpha_{\chi}=-\frac{2}{3} a_{1}^{2} \Delta^{-1} \delta_{v}=\frac{3}{5} C+\frac{4}{9} d t^{-5 / 3}, \\
& v_{\chi}=a_{1} t^{2 / 3} \Delta^{-1} \kappa_{v}=-\frac{1}{a_{1}}\left(\frac{3}{5} C t^{1 / 3}-\frac{2}{3} d t^{-4 / 3}\right) .
\end{aligned}
$$

Notice that to the linear order we have

$$
\varphi_{v}=C
$$

and $\varphi_{v}$ has no decaying mode in expanding phase; this is true considering the presence of the cosmological constant. In fact, to the linear order $\varphi_{v}$ satisfies [8]

$$
\dot{\varphi}_{v}=0 \text {. }
$$

See also Eq. (20) in the second reference of [2]. Ignoring the decaying mode, for $\Lambda=0$, we have

$$
\varphi_{\chi}=\frac{3}{5} \varphi_{v}
$$


and the temperature anisotropy of cosmic microwave background radiation (CMB) gives $[16,17]$

$$
\frac{\delta T}{T} \sim \frac{1}{3} \varphi_{\chi} \sim \frac{1}{5} \varphi_{v} \sim \frac{1}{5} C,
$$

to the linear order. This is a part of the Sachs-Wolfe effect in a flat background without the cosmological constant. The observations of CMB give $\delta T / T \sim 10^{-5}$ [18], thus

$$
\varphi_{v} \sim 5 \times 10^{-5},
$$

in the large-scale limit near horizon scale. Our $\varphi$ is dimensionless.

We call $\varphi$ the curvature perturbation because it is related to the perturbed part of the spatial curvature of the normal hypersurface. To the linear order we have

$$
R^{(h)}=\frac{6 \bar{K}}{a^{2}}-4 \frac{\Delta+3 \bar{K}}{a^{2}} \varphi,
$$

where $R^{(h)}$ is the scalar-curvature of the three-space metric $h_{\alpha \beta}$, and $\bar{K}$ is the sign of the background curvature, see Eq. (4) in [11]. To the second order, see Eq. (265) in [1]. In fact, from Eqs. (7), (55), and (175) of [1] we can easily check that $\varphi$ characterizes the three-space Riemann curvature $R_{\beta \gamma \delta}^{(h) \alpha}$ to all orders in perturbations; this is true assuming pure scalar-type perturbation. The gauge-invariant combination $\varphi_{v}$ is also known to be one of the large-scale (super-sound-horizon) limit conserved variables in various situations including time varying equation of state, field potential, and generalized gravity theories [19]. It is also known to be conserved in the large-scale (super-sound-horizon) limit even in nonlinear situations $[20,1]$.

\section{INCLUDING THE GRAVITATIONAL WAVES}

In this section we present the complete set of equations to the third order, now including the contribution of gravitational waves, and also the gravitational wave equation complete to the second order. Using Eqs. (14), (15), and (24), Eqs. (20), (21), and (23) give

$$
\begin{aligned}
\dot{\delta} & +\frac{1}{a} \nabla \cdot \mathbf{u}=-\frac{1}{a} \nabla \cdot(\delta \mathbf{u}) \\
& +\frac{1}{a}\left[2 \varphi \mathbf{u}-\nabla\left(\Delta^{-1} X\right)\right] \cdot \nabla \delta+\frac{2}{a} \delta^{, \alpha} u^{\beta} C_{\alpha \beta}^{(t)}, \\
\frac{1}{a} \nabla \cdot(\dot{\mathbf{u}}+H \mathbf{u})+4 \pi G \mu \delta & \\
& =-\frac{1}{a^{2}} \nabla \cdot(\mathbf{u} \cdot \nabla \mathbf{u})-\dot{C}^{(t) \alpha \beta}\left(\frac{2}{a} u_{\alpha \mid \beta}+\dot{C}_{\alpha \beta}^{(t)}\right) \\
& -\frac{2}{3 a^{2}} \varphi \mathbf{u} \cdot \nabla(\nabla \cdot \mathbf{u})+\frac{4}{a^{2}} \nabla \cdot\left[\varphi\left(\mathbf{u} \cdot \nabla \mathbf{u}-\frac{1}{3} \mathbf{u} \nabla \cdot \mathbf{u}\right)\right] \\
& -\frac{\Delta}{a^{2}}\left[\mathbf{u} \cdot \nabla\left(\Delta^{-1} X\right)\right]+\frac{1}{a^{2}} \mathbf{u} \cdot \nabla X+\frac{2}{3 a^{2}} X \nabla \cdot \mathbf{u}
\end{aligned}
$$

which generalizes Eq. (28) to include the gravitational waves.

Now, we present the equation for tensor-type perturbation to the second order. From Eq. (103), (210) of [1] we can derive equation for $\ddot{C}_{\alpha \beta}^{(t)}$ to the second order. Since we are ignoring the vector-type perturbation, from Eqs. (211), (199) of [1] we have

$$
\ddot{C}_{\alpha \beta}^{(t)}+3 H \dot{C}_{\alpha \beta}^{(t)}-\frac{\Delta}{a^{2}} C_{\alpha \beta}^{(t)}=N_{4 \alpha \beta}
$$




$$
-\frac{3}{2}\left(\nabla_{\alpha} \nabla_{\beta}-\frac{1}{3} g_{\alpha \beta}^{(3)} \Delta\right) \Delta^{-2} \nabla^{\gamma} \nabla^{\delta} N_{4 \gamma \delta} .
$$

From Eq. (103) of [1] to the second order we have

$$
\begin{aligned}
& N_{4 \alpha \beta}=\frac{1}{a^{3}}\left\{a ^ { 3 } \left[\frac{2}{a^{2}}\left(\varphi \chi_{, \alpha \mid \beta}+\varphi_{,(\alpha} \chi_{, \beta}\right)+2 \varphi \dot{C}_{\alpha \beta}^{(t)}\right.\right. \\
& +\frac{2}{a^{2}} \chi_{\mid \beta}^{, \gamma} C_{\alpha \gamma}^{(t)}+\frac{1}{a^{2}} \chi^{, \gamma}\left(2 C_{\gamma(\alpha \mid \beta)}^{(t)}-C_{\alpha \beta \mid \gamma}^{(t)}\right) \\
& \left.\left.+2 C_{\alpha}^{(t) \gamma} \dot{C}_{\beta \gamma}^{(t)}\right]\right\} \\
& +\frac{1}{a^{4}} \chi_{\mid \alpha}^{, \gamma} \chi_{, \gamma \mid \beta}+\frac{1}{a^{2}}\left(\kappa \chi, \alpha \mid \beta-3 \varphi_{, \alpha} \varphi, \beta-4 \varphi \varphi_{, \alpha \mid \beta}\right) \\
& +\kappa \dot{C}_{\alpha \beta}^{(t)}+\frac{1}{a^{2}}\left[2 \varphi^{, \gamma}{ }_{\mid \alpha} C_{\beta \gamma}^{(t)}-2 \Delta \varphi C_{\alpha \beta}^{(t)}-4 \varphi \Delta C_{\alpha \beta}^{(t)}\right. \\
& +\varphi^{, \gamma}\left(2 C_{\gamma(\alpha \mid \beta)}^{(t)}-3 C_{\alpha \beta \mid \gamma}^{(t)}\right)+2 \chi^{, \gamma}{ }_{\mid[\alpha} \dot{C}_{\beta] \gamma}^{(t)}-\chi^{, \gamma} \dot{C}_{\alpha \beta \mid \gamma}^{(t)} \\
& +2 C^{(t) \gamma \delta}\left(2 C_{\gamma(\alpha \mid \beta) \delta}^{(t)}-C_{\alpha \beta \mid \gamma \delta}^{(t)}-C_{\gamma \delta \mid \alpha \beta}^{(t)}\right) \\
& \left.-2 C^{(t) \gamma} \Delta C_{\beta \gamma}^{(t)}-C^{(t) \gamma}{ }_{\delta \mid \alpha} C^{(t) \delta}{ }_{\gamma \mid \beta}+4 C^{(t) \gamma \mid \delta} C_{\beta[\delta \mid \gamma]}^{(t)}\right] \\
& -\frac{1}{3} g_{\alpha \beta}^{(3)}\left\{\frac { 1 } { a ^ { 3 } } \left\{a ^ { 3 } \left[\frac{2}{a^{2}}\left(\varphi \Delta \chi+\varphi^{, \gamma} \chi_{, \gamma}\right)\right.\right.\right. \\
& \left.\left.+2 C^{(t) \gamma \delta}\left(\frac{1}{a^{2}} \chi_{, \gamma \mid \delta}+\dot{C}_{\gamma \delta}^{(t)}\right)\right]\right\}^{\cdot}+\frac{1}{a^{4}} \chi^{, \gamma \mid \delta} \chi_{, \gamma \mid \delta} \\
& +\frac{1}{a^{2}}\left[\kappa \Delta \chi-4 \varphi \Delta \varphi-3 \varphi^{, \gamma} \varphi_{, \gamma}+2 \varphi^{, \gamma \mid \delta} C_{\gamma \delta}^{(t)}\right. \\
& \left.\left.-4 C^{(t) \gamma \delta} \Delta C_{\gamma \delta}^{(t)}+C^{(t) \gamma \delta \mid \epsilon}\left(2 C_{\gamma \epsilon \mid \delta}^{(t)}-3 C_{\gamma \delta \mid \epsilon}^{(t)}\right)\right]\right\} . \quad(44)
\end{aligned}
$$

In Eq. (44) we have ignored $\alpha$ and $\dot{\varphi}$ terms which are already quadratic order in the comoving gauge. We have $\chi=\chi_{v}, \varphi=\varphi_{v}, \kappa=\kappa_{v}$ and $C_{\alpha \beta}^{(t)}=C_{\alpha \beta v}^{(t)}$ which are gauge-invariant combinations. Apparently, we need $\chi_{v}$, $\kappa_{v}$ and $\varphi_{v}$ to the linear order. To that order, we have identified $\kappa_{v}=-\frac{1}{a} \nabla \cdot \mathbf{u}$ and $\mathbf{u}=\frac{1}{a} \nabla \chi_{v}$. For $\varphi_{v}$ we have Eq. (29). Using these identifications we can express the scalar-type perturbation variables in Eq. (44) in terms of the Newtonian variables.

\section{DISCUSSION}

We have derived the third-order perturbation equations in the zero-pressure cosmological medium in Einstein's gravity. We have expressed the third-order terms using the Newtonian variables identified in the lower order perturbations. Since the Newtonian zero-pressure cosmological medium is exact to the second order in perturbation, our third-order terms in relativistic context are pure relativistic corrections. Our results show that the third-order correction terms in relativistic energy and momentum conservation equations are of $\varphi_{v}$-order higher than the second-order terms, thus equivalently $\varphi_{v}$-order higher than the Newtonian terms. The corrections terms are independent of the horizon scale and depend only on $\varphi_{v}$ to the linear order which is the spatial curvature perturbation in the comoving gauge (hypersurface), or $\sim \delta \Phi$ which is the gravitational potential perturbation strength. The variable $\varphi_{v}$ is known to have conserved behavior and its amplitude in the large-scale (near horizon, say) is constrained by the low-level anisotropies of the CMB temperature, see Eq. (37). Therefore, our result reinforces our previous conclusion in [2] that one can use the large-scale Newtonian numerical simulation more reliably even as the simulation scale approaches near (and goes beyond) the horizon.

In this work we have assumed a single zero-pressure irrotational fluid in the flat cosmological background. Dropping any of these conditions could potentially lead to relativistic corrections. Due to these assumptions we cannot apply our results when the radiation components (including neutrino anisotropic stress) become important in high redshift epoch, and in the case when the baryon generated entropy leads to rotational perturbations in the small-scale clusters. ${ }^{*}$ Extensions to include relativistic second-order perturbational effects of the pressure, the rotation, the non-flat background, and the multicomponent situation will be investigated in future occasions. In this work we derived the relativistic correction terms appearing in the third order, and showed that these correction terms do not involve the horizon scale and are small in our observable patch.

Besides theoretical and practical significance we believe our exact result to the second order and pure relativistic corrections to the third order have historical value as well, because these have been unsolved issues since Lifshitz's original work in the linear regime in 1946 [5]. Before our present third order and the previous second order works there were different anticipations among researchers in the field that even in the second order the relativistic result might be different from the Newtonian ones: one common anticipation was that the general relativistic effects might become important as the scale approaches and goes beyond the horizon. Our results resolved such an issue and showed that there exist no correction terms to the second order in all scales. We also showed that pure relativistic correction terms appearing in third order do not depend on the horizon scale. It depends only on the strength of the dimensionless gaugeinvariant curvature perturbation variable $\varphi_{v}$ or the dimensionless linear-order gravitational potential $\delta \Phi$.

The post-Newtonian approximation [21] provides a complementary method to our perturbative approach in deriving the relativistic correction terms in the New-

\footnotetext{
${ }^{*}$ We thank the anonymous referee for making this point.
} 
tonian cosmology. The post-Newtonian approximation takes $v / c$-expansion with $v / c \ll 1$, thus for near virialized systems we have $G M /\left(R c^{2}\right) \sim v^{2} / c^{2} \ll 1$. Thus, although such an approximation takes into account of nonlinearity it is valid only far inside the horizon; as we approach the horizon $G M /\left(R c^{2}\right)$ becomes unity. Consistency of the Newtonian cosmology with the Newtonian limit of the post-Newtonian approximation was reported in [22]. We can show that the Newtonian cosmological hydrodynamic equations naturally appear in the zeroth-order post-Newtonian approximation [23]. Recently, we derived the fully nonlinear first-order postNewtonian correction terms, and showed that these correction terms have typically $G M /\left(R c^{2}\right) \sim v^{2} / c^{2} \sim 10^{-5}$ order smaller than the Newtonian terms in the nonlinearly clustered regions [23]. Being a complimentary approach to the post-Newtonian approximation (which provides fully non-linear equations) our equations valid to the third-order perturbations may have diverse applications in the cosmological situations where the systems have not reached fully nonlinear stage. Compared with the post-Newtonian approach, our perturbation approach is applicable in all cosmological scale.

Even in the small (far less than the horizon) scale the pure (third-order) relativistic correction terms could have important roles if the strength of linear-order $\varphi_{v}$ is large enough. Our relativistic results are valid in the perturbative sense. Thus, if $\varphi_{v}$ approaches near unity, higherorder perturbative terms could become important as well, and it is likely that our perturbative approach breaks down. Still, it would be interesting to investigate regimes where $\varphi_{v}$ is moderately important so that we can study the roles of pure relativistic effects using our third-order correction terms. For example, whether such pure relativistic correction terms could lead to an observationally distinguishable non-Gaussian signature [24] is an interesting issue which may deserve further attention. For such investigations, Eqs. (25)-(28) are the complete set for pure scalar-type perturbation, and Eqs. (39)-(44) provide the complete set including the gravitational waves. As we consider a flat background the ordinary Fourier analysis can be used to study the mode-couplings as in the Newtonian case in [25]. Comparing the roles of pure relativistic third-order corrections with the inherent third-order perturbation effects in the Newtonian approximation in Eqs. (25)-(28) will be an interesting step we can take. Applications to such cosmological situations are left for future studies.

\section{Acknowledgments}

HN and JH were supported by grants No. R04-200310004-0 and No. R02-2003-000-10051-0, respectively, from the Basic Research Program of the Korea Science and Engineering Foundation.
[1] H. Noh and J. Hwang, Phys. Rev. D 69, 104011 (2004).

[2] H. Noh and J. Hwang, Class. Quant. Grav. 22, 3181 (2005); J. Hwang and H. Noh, Phys. Rev. D 72, 044011 (2005).

[3] A.A. Friedmann, Zeitschrift für Physik 10, 377 (1922), and ibid. 21, 326 (1924); both papers are translated in Cosmological-constants: papers in modern cosmology, edited by J. Bernstein and G. Feinberg (Columbia Univ. Press, New York, 1986), p49 and p59; H.P. Robertson, Proceedings of the National Academy of Science 15, 822 (1929).

[4] E.A. Milne, Quart. J. Math. 5, 64 (1934); W.H. McCrea and E.A. Milne, ibid. 5, 73 (1934).

[5] E.M. Lifshitz, J. Phys. (USSR) 10, 116 (1946).

[6] W.B. Bonnor, Mon. Not. R. Astron. Soc. 117, 104 (1957).

[7] P.J.E. Peebles, The large-scale structure of the universe, (Princeton Univ. Press, Princeton, 1980).

[8] J. Hwang and H. Noh, Gen. Rel. Grav. 31, 1131 (1999).

[9] D.N. Spergel, et al., Astrophys. J. Suppl. 148, 175 (2003); M. Tegmark, et al., Phys. Rev. D 69, 103501 (2004).

[10] J.M. Bardeen, Particle Physics and Cosmology, edited by L. Fang and A. Zee (Gordon and Breach, London, 1988), p1.

[11] J. Hwang, Astrophys. J. 375, 443 (1991).

[12] J. Ehlers, Proceedings of the mathematical-natural science of the Mainz academy of science and literature, Nr. 11, 792 (1961), translated in Gen. Rel. Grav. 25, 1225 (1993); G.F.R. Ellis, in General relativity and cosmology, Proceedings of the international summer school of physics Enrico Fermi course 47, edited by R. K. Sachs (Academic Press, New York, 1971), p104; in Cargese Lectures in Physics, edited by E. Schatzmann (Gorden and Breach, New York, 1973), p1.

[13] R. Arnowitt, S. Deser, and C.W. Misner, in Gravitation: an introduction to current research, edited by L. Witten (Wiley, New York, 1962) p. 227.

[14] J.M. Bardeen, Phys. Rev. D 22, 1882 (1980).

[15] J. Hwang Astrophys. J. 427, 533 (1994).

[16] R.K. Sachs and A.M. Wolfe, Astrophys. J. 147, 73 (1967).

[17] J. Hwang and H. Noh, Phys. Rev. D 59, 067302 (1999).

[18] G.F. Smoot, et al. Astrophys. J. 396, L1 (1992).

[19] J. Hwang, Phys. Rev. D 53, 762 (1996); J. Korean Phys. Soc. 35, S633 (1999); J. Hwang and H. Noh, Phys. Rev. D 61, 043511 (2000); ibid. 66, 084009 (2002); ibid. 71, 063536 (2005).

[20] D.S. Salopek and J.R. Bond, Phys. Rev. D 42, 3936 (1990).

[21] S. Chandrasekhar, Astrophys. J. 142, 1488 (1965).

[22] E. Bertschinger and A.J.S. Hamilton, Astrophys. J. 435 (1994) 1; L. Kofman and D. Pogosyan, ibid. 442 (1995) 30; G.F.R. Ellis and P.K.S. Dunsby, Astrophys. J. 479, 97 (1997). 
[23] J. Hwang, H. Noh, and D. Puetzfeld, Phys. Rev. D submitted (2005), astro-ph/0507085.

[24] F. Bernardeau, S. Colombi, E. Gaztanaga, and R. Scoccimarro, Phys. Rep. 367, 1 (2002); N. Bartolo, E. Komatsu, S. Matarrese, and A. Riotto, Phys. Rep. 402, 103 (2004).

[25] E.T. Vishniac, Mon. Not. R. Astron. Soc. 203, 345 (1983); M.H. Goroff, B. Grinstein, S.-J. Rey, and M.B. Wise, Astrophys. J., 311, 6 (1986). 